\title{
Glassy Dynamics of Polystyrene by Quasielastic Neutron
}

\section{Scattering}

S. Arrese--Igor ${ }^{1}$, A. Arbe ${ }^{1}$ B. Frick ${ }^{2}$ J. Colmenero ${ }^{1,3,4}$

1 Centro de Física de Materiales (CSIC-UPV/EHU),

and Materials Physics Center MPC Paseo Manuel de Lardizabal 5, 20018 San Sebastián, Spain

2 Institut Laue-Langevin, BP 156X, 38042 Grenoble Cedex 9, France

3 Departamento de Física de Materiales UPV/EHU,

Paseo Manuel de Lardizabal 3, 20018 San Sebastián, Spain and

4 Donostia International Physics Center,

Paseo Manuel de Lardizabal 4, 20018 San Sebastián, Spain*

(Dated: March 7, 2011)

Neutron scattering measurements of partially deuterated polystyrene (PS) samples

- PSd3 with deuterated main chain and PSd5 with deuterated phenyl rings - have been carried out below the glass transition temperature $\left(T_{g}=373 \mathrm{~K}\right)$ of the polymers by means of time-of-flight (TOF) and backscattering techniques (BS). Analysis of the data was performed putting emphasis on slow relaxation motions at $t>2 p s$. Results show that hydrogens in phenyl rings have higher mobility than those in the main chain. The mean square displacement, $\left\langle u^{2}>\right.$, of the main chain on the other hand, is almost indistinguishable from those reported for chemically simpler polymers. The intermediate scattering functions obtained by Fourier transformation of experimental data in the TOF window indicate than $\left\langle u^{2}\right\rangle$ is mainly due to motions faster that $\sim 2 p s$, and that secondary relaxations have little effect on its 
value. In contrast to previous observations in several polymers containing phenyl rings in the main chain, rings in PS do not exhibit generalized $180^{\circ}$ flips, and the quasielastic scattering of phenyl rings in PS at $t>2 p s$ (including the BS window) can be reasonably well described by oscillations of increasing amplitude with temperature and mean activation energy of $0.21 \mathrm{eV}$. Our results support those NMR works which claim a low fraction of flipping rings for PS below $T_{g}$, and low amplitude motions for the origin of $\delta$ and $\gamma$-relaxations. 


\section{INTRODUCTION}

The origin of secondary relaxations in polymers is still not well understood in spite of the great effort made over more than 20 years. The interest in the study of these processes is two fold: on the one hand, from a technological point of view, correlations have been proposed between the ductile-fragile transition temperature and that of secondary relaxation; on the other hand, the basic understanding of the dynamic processes in polymers is a question of topmost interest. It is worth emphasizing that, apart from a few exceptions, almost all the dynamic studies concerning this question have been carried out by means of relaxation techniques (dielectric, mechanical,..) which, in general, do not cover the high-frequency part of the spectrum and do not provide microscopic spatial information about the motion involved. In a complementary way to the existing NMR studies, it is clear that neutron scattering (NS) techniques can shed new light on this problem.

Previous experiences in this direction have shown that the combination of several NS instruments gives the opportunity to obtain detailed characterization of the sub- $T_{g}\left(T_{g}\right.$ : glass-transition temperature) motions in general and of phenyl-ring motions in particular. The later have, for example, been extensively studied in a series of engineering thermoplastics containing the bisphenol-A unit, namely polysulfone $(\mathrm{PSF})^{1}$, polycarbonate $(\mathrm{PC})^{2}$, phenoxy $(\mathrm{PH})^{3}$, and the polymer membrane polyethersulfone $(\mathrm{PES})^{4}$. In this work we explore the sub- $T_{g}$ dynamics in polystyrene (PS). PS is a well-known polymer with plenty of applications but also frequently used as a model polymer for fundamental research. In contrast to the previous polymers mentioned, phenyl rings in PS are not located in the main chain but they are a side group themselves. The position of a molecular group either in the main chain or in the side chain has often been considered to be decisive for the role and relevance of its 
motion with respect to the mechanic properties and the glass $\operatorname{transition}^{5-8}$.

The study of PS's sub- $T_{g}$ dynamics by relaxation techniques has been extensive. Mechanic and dielectric spectroscopy (MS and DS) experiments show that PS exhibits several secondary relaxations named $\beta, \gamma$, and $\delta^{9}$. NMR work generally agrees with the presence of ring motions extending over several orders of magnitude in time, and quite restricted spatial motion of the main chain below $T_{g}$. However, the interpretations in detail vary from work to work ${ }^{10-14}$. A common characteristic of these NMR models is to separate the rings into a "slow" and a "fast" fraction. Most authors relate the motion of "fast"10,11, and "slow"11,12 rings to the $\gamma$ and $\beta$ relaxations respectively, and propose a coordinated motion for main chain and phenyl rings ${ }^{10,13}$. The motion of the main chain as seen by NMR on the other hand, is reported to be highly restricted (less than $\left.1^{o}\right)^{13,14}$. The dynamics of PS has also been simulated by various authors ${ }^{15-19}$. Suter et al. found by means of quasistatic simulations that the frequency distribution for the ring motion covers many orders of magnitude and that small reorientation of phenyl rings dominate the dynamics below $\mathrm{T}_{g}{ }^{15}$. The Molecular Dynamics Simulations (MDS) of Lyulin et al. ${ }^{17}$ below $T_{g}$ show for both, phenyl rings and main chain, translational and rotational motions whose characteristic times correspond quite well to those reported for $\gamma$ and $\beta$ relaxations respectively (Fig.1). The dynamics of phenyl rings and main chain had very similar characteristic times and were considered correlated due to excluded-volume interactions. In spite of all these experimental efforts, the compilation of the information in the literature is not fully consistent and raises several questions. (i) First, the fraction of flipping (180 rotations) rings found by NMR varies significantly from work to work: $7 \%^{10}, 20 \%^{14}, 82 \%^{12}, 100 \%^{11}$. In simulations Suter et al. ${ }^{15}$ found this fraction to be less than $3 \%$, and Lyulin et al. ${ }^{18}$ found $2 \%$ of flipping rings after a 24 ns run. (ii) Second, the various interpretations for the different processes are somehow 
contradictory. The molecular origin of the $\gamma$-relaxation for which the characteristic time is shown in Fig.1 has been assigned either to ring flips (rectangular area ${ }^{10}$ and big circle $^{11}$ ), ring oscillations (big triangle ${ }^{12}$ ), or to ring and main-chain translation motions (solid circles and squares $\left.{ }^{17}\right)$. Likewise, the motions around the spectroscopic $\beta$-relaxation in the same figure have been assigned to ring's small angle rotations (rectangular area ${ }^{10}$ ), ring flips plus small angle rotations (up-triangles ${ }^{12}$ ), and ring flips (empty circles ${ }^{11}$ ).

We aimed to clarify the molecular origin of the relaxation map of sub-Tg motions in PS and to obtain information about main-chain and phenyl-ring dynamics by combining the NS techniques time of flight (TOF) and backscattering (BS) on selectively deuterated samples. Incoherent quasielastic NS offers a unique opportunity to check the models for different "moving" ring fractions as the ratio of the elastic to quasielastic intensity depends among others on the number of moving hydrogens. Previous neutron scattering studies of PS in the literature ${ }^{21-24}$ were either focused on the study of TOF data in the frequency domain (mainly the so called "fast-dynamics"), did not fully exploit H/D labeling capabilities or did not provide full analysis of the quasielastic signal in BS. The emphasis here is more oriented to slower relaxation motions below $T_{g}$, and thereby in the present work: (i) TOF data are Fourier transformed to the time domain in order to separate fast contributions $(t<2 p s)$ from slower ones; (ii) very high resolution BS instrument is used in order to extend the study to slower times; and (iii) analysis of the quasielastic BS signal is provided. To our knowledge there is no precedent combining the former three points for both phenyl-ring deuterated and main-chain deuterated samples in the same work. The paper is organized as follows. After describing some experimental details in section II, results will be presented in section III divided in two different subsections: TOF timescale and BS timescale. Results are discussed throughout section IV. Previous data of phenyl-ring containing polymers, 
in particular of polysulfone with deuterated methyl groups $(\mathrm{PSFd} 6)^{1}$ will be considered as reference for discussion. Finally, conclusions are exposed in section V.

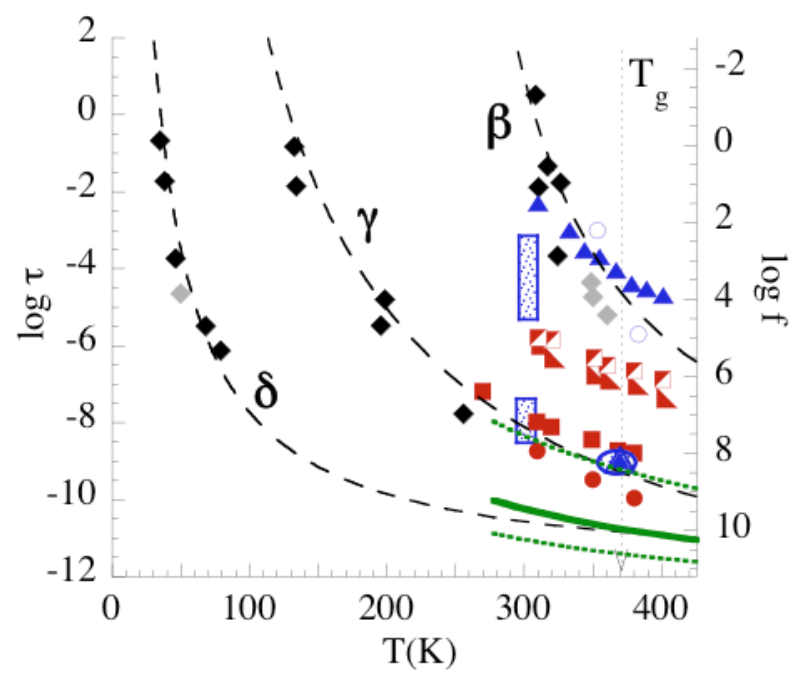

FIG. 1: Characteristic times map for different dynamic processes in PS measured by different techniques: (i) Mechanic and dielectric relaxations, filled and empty diamonds respectively ${ }^{9}$; (ii) NMR, up-triangles ${ }^{12}$, empty circles ${ }^{11}$ and shadowed rectangular areas ${ }^{10}$; (iii) MDS, solid circles, solid and half-solid squares, and right-triangles ${ }^{17}$; and (iv) NS this work, solid and dotted lines. Dashed lines represent fits of data in the literature to Arrhenius laws.

\section{EXPERIMENTAL DETAILS}

Quasielastic NS measurements were carried out in two dynamic windows by means of the IN6 time of flight (TOF) and the IN16 backscattering (BS) spectrometers at the Institute Laue Langevin (ILL) covering timescales from $\simeq 10^{-13}$ to $10^{-11} s$ and from $\simeq 10^{-10}$ to $10^{-9} s$ respectively. Flat samples in aluminum containers were used and their thickness was adjusted to obtain a transmission coefficient close to $90 \%$ thus reducing multiple scattering events for which we have not apply any correction. Raw data were corrected for detector effi- 
ciency, sample container, and self-absorption. Additionally, time of flight to energy transfer $(\hbar \omega)$ and scattering angle to momentum transfer $(Q)$ interpolations were done in the case of TOF measurements. For TOF measurements on IN6 an incident wavelength of $5.12 \AA$ was used resulting in a resolution with half width at half maximum (HWHM) of about $43 \mu \mathrm{eV}$. The scattering angle $\theta$ extended from $11.5^{\circ}$ to $113.5^{\circ}$ and the energy window ranged from $\sim-2 \mathrm{meV}$ to some hundreds of $\mathrm{meV}$. After angle to $Q$ interpolation the constant $Q$ curves cover the range $0.32 \leq Q \leq 2 \AA^{-1}$. For backscattering measurements an incident wavelength of $6.271 \AA$ was used, covering a $Q$ range between 0.2 and $1.9 \AA^{-1}$ and an energy window from -15 to $15 \mu \mathrm{eV}$ with $0.45 \mu \mathrm{eV}$ (HWHM) resolution.

Commercially available (Polymer Source) atactic and partially deuterated PS samples with deuterated main chain (PSd3) and deuterated phenyl rings (PSd5) have been studied. The molecular weight and polydispersity of the samples were $M_{w}=26800$ and PI=1.12 for PSd3, and $M_{w}=19500$ and PI=1.08 for PSd5. The glass transition temperatures for the samples measured by differential scanning calorimetry at $20 \mathrm{~K} / \mathrm{min}$ rate were $365 \mathrm{~K}$ for PSd3 and 375 for PSd5. Both samples were measured by IN16 and IN6 instruments: i) PSd3 on IN16: at 10, 300,340, 367 and $400 \mathrm{~K}$ for $\sim 10$ hours and short runs of $\sim 1.3$ hours at 50,100, 150, 200, and $250 \mathrm{~K} . P S d 3$ on IN6: at 10, 100, 200, 250, 300, 340 and $368 \mathrm{~K}$ for $\sim 2.6$ hours; ii) PSd5 on IN16: at 10,340, 365 and $396 \mathrm{~K}$ for $\sim 9.5$ hours and short runs of 1.3 hours at 100, 200, 250, and 300K. PSd5 on IN6: at 13, 200, 250, 300, 340 and $368 \mathrm{~K}$ for $\sim 2.6$ hours. The incoherent scattering cross section of hydrogen is much higher than those of the rest of the atoms present in the samples. Accordingly, diffraction measurements with polarization analysis ${ }^{25}$ show that for both samples the coherent intensity is much weaker than the incoherent one (see Fig.3). As a consequence, by NS on PSd3 we address mainly the motion of hydrogens in phenyl rings, while measurements on PSd5 access essentially 
that of hydrogens in the main chain.



FIG. 2: PSd5 (panel a) and PSd3 (panel b) spectra measured by IN6-TOF at $\mathrm{Q}=1.37 \AA^{-1}$ and at different temperatures 10, 100 (only PSd3), 200, 250, 300 ,340 and $368 \mathrm{~K}$ from bottom to top. Inset: detail of the elastic intensity. 


\section{RESULTS}

\section{A. Time Of Flight}

Figure 2 shows spectra obtained for PSd5 and PSd3 at $\mathrm{Q}=1.37 \AA^{-1}$ and at various temperatures below $T_{g}$. The Boson-peak characteristic of glass forming systems can be identified at energies $\sim 1-1.5 \mathrm{meV}$. As the temperature increases, the elastic intensity decreases and a quasielastic contribution increases smearing out the Boson-peak.

The inset in figure 4 shows a detail of the decrease of the elastic intensity with temperature for PSd5 (circles) and PSd3 (squares) at $Q=1.64 \AA^{-1}$. The Q-dependence of the intensity at the elastic maxima normalized to its value at $10 \mathrm{~K}$ (PSd5) or 13K (PSd3) is represented in figures 3a) and b) for PSd3 and PSd5 respectively. The ratio of coherent to total scattered intensities ${ }^{25}$ has also been included on the r.h.s.-axis of figure 3 (crosses). The $Q$-dependence of this quantity is determined by the partial structure factor corresponding to the H/D labeling of the sample investigated. After normalization to the low-T value the elastic intensity presents a very slight modulation attributable to the T-dependence of the corresponding partial structure factor. Under the Gaussian approximation, the fit of data in figures 3 a) and b) to $\propto \exp \left[-Q^{2}<u^{2}>/ 3\right]$ (lines), leads to the mean squared displacement $<u^{2}>$ plotted in figure 4 . Filled symbols represent data from long isothermal measurements and empty symbols from ten-minutes runs during heating or cooling. The mean square displacement for reference temperatures (10 and 13K) were taken equal to zero. At low temperatures, $\left\langle u^{2}>\right.$ follows the linear T-dependence characteristic for harmonic vibrations. Solid lines in figure 4 represent linear fits of the $\left\langle u^{2}\right\rangle$ for the somewhat arbitrary chosen limit of $\mathrm{T} \leq 150 \mathrm{~K}$.

For PSd3 figure 5 shows the experimental intermediate scattering function $S_{\text {exp }}(Q, t, T)$ 

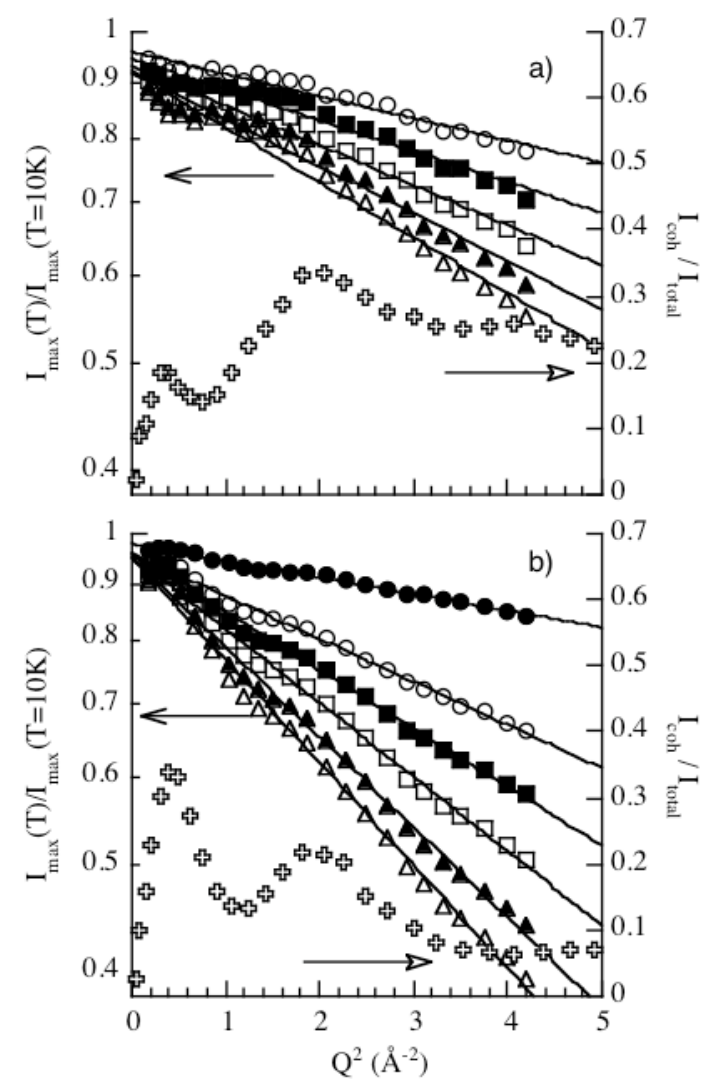

FIG. 3: Elastic intensity of PSd5 (panel a) and PSd3 (panel b) in TOF window normalized to 10 $\mathrm{K}$ and $13 \mathrm{~K}$ values respectively. Solid lines represent fittings to $\propto \exp \left[-<u^{2}>Q^{2} / 3\right]$. Crosses represent the ratio of coherent to total intensity measured by diffraction measurements at $\mathrm{D} 7^{25}$.

$=I_{\text {exp }}(Q, t, T) / I_{\text {exp }}(Q, t, T=10 K)$ obtained by Fourier Transform of the experimental spec$\operatorname{tra} I_{\text {exp }}(Q, \omega)$. This representation is very useful as it allows direct deconvolution of the instrumental resolution by simple division and, as we will see later, helps to interpret the origin of the quasielastic broadening and the non-harmoniticy effects looking at their timescale. The $S_{\text {exp }}(Q, t, T)$ curves show a relatively pronounced decay at times shorter than $1-2 p s$ while the decay at $t \gtrsim 2 p s$ is either almost zero at low temperatures or very small at the highest ones. This kind of crossover at about $1-2 p s$ separating fast and slow relaxation regimes is common in polymeric systems ${ }^{1,2,19,26,27}$ and usually evolves to a clear two step 


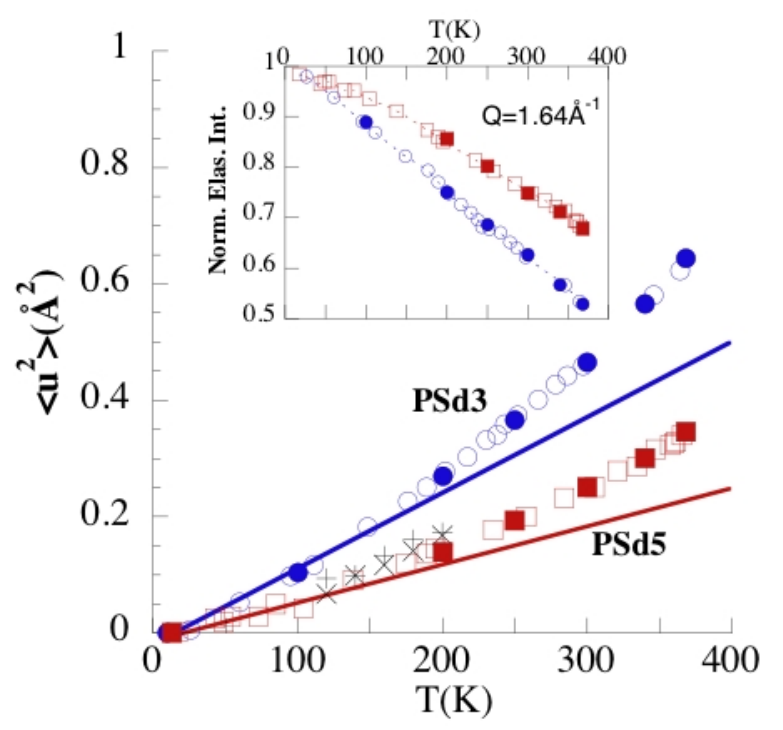

FIG. 4: Mean squared displacement as a function of temperature for PSd5 (squares) and PSd3 (circles) obtained from the elastic maxima of the spectra in TOF window (see text). Solid lines represent fits to an harmonic law at $T \leq 150 \mathrm{~K}$. Plusses and crosses represent the $<u^{2}>$ of Polyethylenepropylene and Head to head Polypropylene respectively ${ }^{28}$. Inset: T-dependence of the elastic intensity normalized to $10(\mathrm{PSd} 3)$ and $13 \mathrm{~K}$ (PSd5) values at $Q=1.64 \AA^{-1}$.

decay when the slower relaxation is fast enough to enter the experimental window. The experimental intermediate scattering functions qualitatively agree those obtained by MDS by Roe in reference ${ }^{19}$. Due to the small decay observed in the $S_{\text {exp }}(Q, t, T)$ function at $t \gtrsim 2 p s$, by simple inspection we can say that the increase of hydrogens' $<u^{2}>$ with temperature is mainly due to motions faster than $\sim 2$ ps. Accordingly, the set of $<u^{2}>_{2 p s}$ values obtained by fitting the Q-dependence of the intermediate scattering function at $2 p s$, i.e. fitting $S_{\exp }(Q, t=2 p s, T) / S_{\exp }(Q, t=2 p s, T=10 K)$ to $\exp \left[-Q^{2}<u^{2}>_{2 p s} / 3\right]$, result to be almost indistinguishable from the $\left\langle u^{2}\right\rangle$ values shown in figure 4 , which were obtained in the frequency domain from $I_{\max }(Q, \omega, T) / I_{\max }(Q, \omega, T=10 K)$. 
Let's now focus on the weaker decay observed at times longer than 2 ps. The behaviour and shape of the $S_{\exp }(Q, t, T)$ functions in this range is very similar to that observed for the phenyl rings in PSF, PES, PH or PC below their respective $T_{g} \mathrm{~s}^{1-4}$. Therefore we have followed here the same data analysis procedure developed in our previous work (see reference ${ }^{1}$ for details). We have calculated the average derivative of the intermediate scattering function between 2 and 20ps for different $T$ - and $Q$-values

$$
A(Q, T)=\left|d S_{\exp }(Q, t) / d \log t\right| .
$$

This magnitude contains features of the quasielastic component of the scattering function and provides important and useful information about the geometry and characteristic time of the motion involved (see references ${ }^{1,2,4}$ for details). For instance, the T-dependence of $A(Q, T)$ gives us hints about the onset of a motion on a particular time scale. Figure 6a shows $A(Q, T)$ for Q-values between 1.5 and $1.6 \AA^{-1}$ as a function of T. $A(Q, T)$ takes significant values at $\mathrm{T} \gtrsim 200 \mathrm{~K}$ indicating that some dynamic process becomes fast enough to produce a small but measurable decay of the intermediate scattering function at $t \gtrsim 2 p s$ from that temperature on. The $Q$-dependence of $A(Q, T)$ revealing spatial information is determined by the features of both the slow motion entering the experimental window at $\mathrm{T} \gtrsim 200 \mathrm{~K}$ and the faster processes producing the decay of the intermediate scattering function at $t \lesssim 2 p$. The effect of the later has been removed dividing $A(Q, T)$ by an effective Debye-Waller factor defined as $D W F_{e f f}=S_{\text {exp }}(Q, t=2 p s, T)$, which was directly read off the experimental intermediate scattering function. The Q-dependence of the $A(Q, T) / D W F_{\text {eff }}$ function now is representative of the geometry of the slow motion producing decay of the $S_{\exp }(Q, t, T)$ at $t \gtrsim 2 p s$. When dealing with incoherent signals, the magnitude $A(Q, T) / D W F_{\text {eff }}$ is proportional to [1-EISF(Q)], where EISF(Q) stands for the Elastic Incoherent Structure Factor ${ }^{20}$ of the local molecular motion causing the slow decay. The $\operatorname{EISF}(\mathrm{Q})$ factor is characteristic for 
a particular geometry of the motion. It is an oscillating function (a combination of Bessel functions in general) with an average amplitude defined by the number of sites visited by the scatterer and maxima and minima positions defined by the distance between sites. During a $180^{\circ}$ flip of phenyl ring for example, hydrogens jump between two equivalent positions 4.3 $\stackrel{\circ}{A}$ apart, so that $[1-\operatorname{EISF}(\mathrm{Q})]$ function presents one maximum at $\mathrm{Q} \sim 1 \AA^{-1}$. Jumps between two equivalent positions separated by $1 \stackrel{\circ}{A}$ or $2 \stackrel{\circ}{A}$, on the other hand show maxima in the [1$\operatorname{EISF}(\mathrm{Q})]$ function at $\mathrm{Q} \sim 4.5$ and $2.2 \AA^{-1}$, respectively. The smaller the displacement of the scattering molecular unit, the larger the $\mathrm{Q}$ value where the [1-EISF $(\mathrm{Q})]$ function presents its first maximum. The $A(Q, T)$ magnitude represented in figure 6 corresponding to the PSd3 samples shows no maxima in the explored Q-range, therefore indicating a small displacement of the hydrogen in phenyl rings.

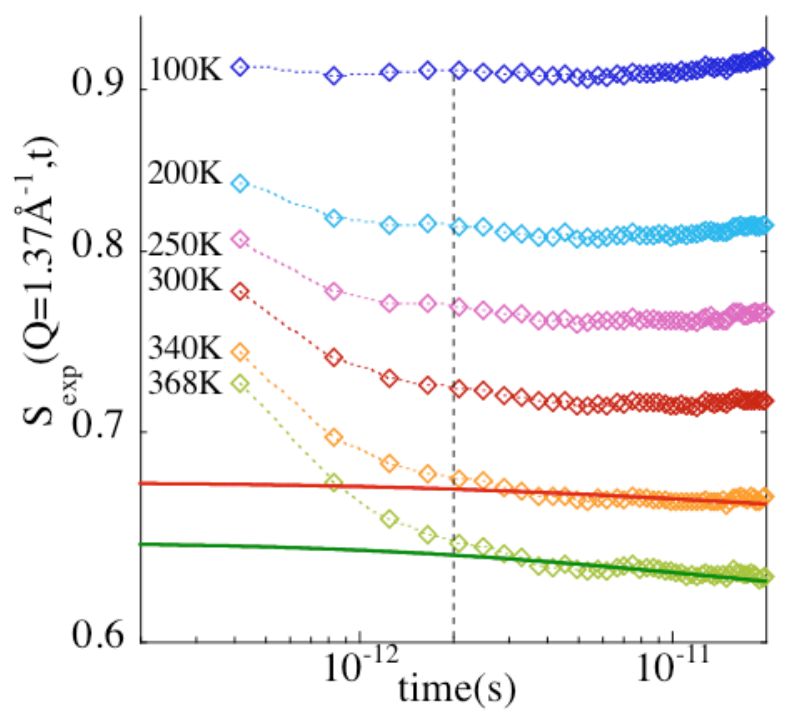

FIG. 5: Intermediate scattering functions for PSd3 obtained by Fourier transform of experimental data measured on IN6 at $\mathrm{Q}=1.37 \AA^{-1}$ and at the temperatures indicated. Lines represent calculated intermediate scattering functions for the oscillation model (see text). Vertical line marks $t=2 \mathrm{ps}$.

In the case of PSd5 sample several factors contribute to make the analysis of the interme- 
diate scattering function at $t>2 p s$ in terms of $A(Q, T)$ less afordable. First, the decay of the intermediate scattering function for PSd5 above $2 p s$ is even smaller than for PSd3 sample. Second, in the most favorable $Q$ range to observe some decay of the intermediate scattering function (between 1.5 and $2.0 \AA^{-1}$ ) the coherent contribution ranges between 25 and $30 \%$. As a result, the $[1-\operatorname{EISF}(\mathrm{Q})]$ function may be no longer describe well the modulation of the quasielastic intensity.
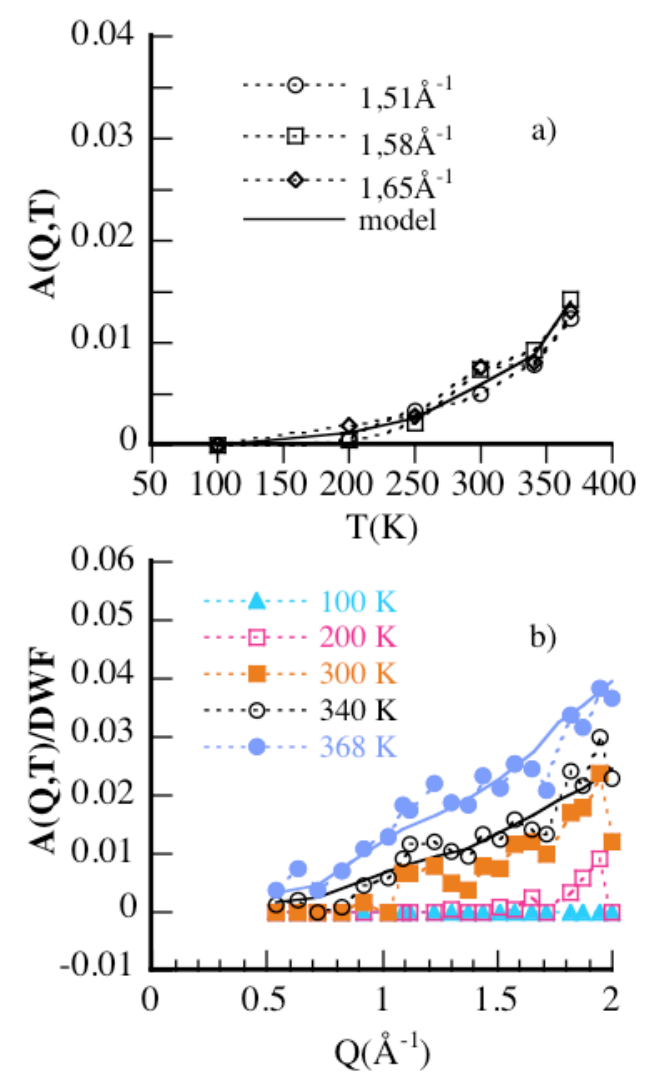

FIG. 6: $A(Q, T)$ and $A(Q, T) / D W F_{\text {eff }}$ for PSd3 in TOF window as a function of T panel a)and Q panel b) respectively. Solid lines represent model calculations (see text). Although the amplitude of the motion is small, it does not show the typical $Q^{2}$ dependence because the model EISF was that resulting from a Gaussian distribution of amplitudes. 


\section{B. Backscattering}

Figure 7 shows the normalized scattering function $I_{\exp }(Q, \omega)$ measured in backscattering (IN16) for PSd3 below $T_{g}$ at 10 and $368 \mathrm{~K}$ (panel a) and above $T_{g}$ at $400 \mathrm{~K}$ (panel b), and for PSd5 at $368 \mathrm{~K}$ (panel c). For comparison results of PSFd6 sample (methyl group deuterated) have also been included in panel b. Although quasielastic broadening above the resolution level is evident in PSd3 for temperatures close to $T_{g}$, this is weaker than that observed for phenyl rings in PSFd6, as the scattering observed at $350 \mathrm{~K}$ in PSFd6 (i.e., 70 $\mathrm{K}$ below PSF's $T_{g}$ ) is of the order of that observed at $400 \mathrm{~K}$ in PSd3 (i.e., $30 \mathrm{~K}$ above PS's $T_{g}$ ) (see panel b of figure 7). On the other hand, as shown in panel $\mathrm{c}$, the quasielastic scattering of main-chain hydrogens in BS is very weak and can be hardly distinguished from the resolution level.

Due to the usually low values of the quasielastic intensity below $T_{g}$ in the backscattering window, an analysis involving integrated intensities is often more suitable. Figures 8 and 9 show the quasielastic intensity integrated for absolute values of the energy transfer between 2 and $5 \mu \mathrm{eV}^{31}$ as a function of $\mathrm{Q}$ and $\mathrm{T}$ respectively. In analogy to the data analysis in the TOF window, this representation of the integrated quasielastic intensity comprises geometric and dynamic information in an intuitive way (see references ${ }^{1,2,4}$ for details). Once the Qdependence of faster motions has been removed (this time dividing by the intensity integrated in the whole window), the quasielastic intensity should follow the modulation imposed by the [1-EISF(Q)] function of the motion (see figure 8). In contrast to the phenyl rings in PSFd6

for example, the quasielastic scattering in PSd3 does not show a clear maximum at $\mathrm{Q} \sim 1 \AA^{-1}$ characteristic for $180^{\circ}$ flips (dashed line in figure 8). Apart from the overall tendency several factors contribute to the small fluctuations of the integrated intensity with Q: i) the poor 




FIG. 7: Normalized scattering functions $I_{\text {exp }}(Q, \omega, T)$ at $Q=1.64 \AA^{-1}$ for PSd3 at $368 \mathrm{~K}$ (panel a) and $400 \mathrm{~K}$ (panel b), and for PSd5 at $368 \mathrm{~K}$ (panel c). Lines stand for measurements at $10 \mathrm{~K}$ representing the resolution of the instrument. Empty symbols in panel b represent data for PSFd6 at $350 \mathrm{~K}$ measured at the same instrument and configuration. 
statistics of data due to the low quasielastic intensity; ii) a weak Q-dependence of the wings of the resolution fuction; and iii) a slight Q-modulation in the total integrated intensity due to coherent contributions. As it is expected, these fluctuations decrease at higher temperatures where the quasielastic contribution is higher. Regarding the T-dependence of the integrated quasielastic intensity, see figure 9 , it is very close to the resolution level and only clearly departs from it at high temperatures, in agreement with spectra in figure 7.

\section{DISCUSSION}

\section{A. TOF and motions $\mathrm{t}<\mathbf{2 p s}$}

As shown in figure 4 , the $\left\langle u^{2}>\right.$ is notably higher for PSd3 than that for PSd5 at all temperatures, reflecting that hydrogens located in phenyl rings (PSd3) perform larger displacements than main-chain hydrogens (PSd5). The results on $<u^{2}>$ of PSd5 obtained in this work agree well with those obtained by Frick at al. and Kanaya et al. for the same deuteration and $1-8 \mu \mathrm{eV}$ and $20 \mu \mathrm{eV}$ resolutions respectively. Consistently, the $<u^{2}>$ for fully protonated PSh in references ${ }^{23,24}$ stays between those for PSd3 and PSd5 measured in this work. The mentioned agreement is good in spite of the different resolutions because $<u^{2}>$ values are dominated by motions faster than $2 p s$. On the other hand, the mobility of main-chain hydrogens is quite similar to that of polymers with sequences of $\mathrm{CH}_{2}$ and $\mathrm{CH}$ unities along their main-chain structure, like polyethylenepropylene and head to head

polypropylene. The $<u^{2}>$ of these two polymers ${ }^{28}$ are compared with that of PSd3 samples by crosses and plusses respectively in figure 4 .

The comparison between the decrease of the elastic intensity of PSd3 and PSd5 has allowed us to better distinguish differences for main-chain and phenyl-ring hydrogen motion 
at low temperatures. In the PSh - PSd5 data sets of reference ${ }^{24}$ the elastic intensities for these two deuterations almost coincide at $\mathrm{T}<200 \mathrm{~K}$, so that similar dynamics could be inferred for main chain and phenyl rings at low temperatures. In contrast, the inset of figure 4 shows that this is not the case when comparing PSd3 and PSd5 data, which shows the difference between the motion at the phenyl rings and main chain, even for very low temperatures.

In principle, the non-linear increase of $\left\langle u^{2}>\right.$ at higher temperatures could be attributed to the onset of anharmonic vibrations, the so-called "fast-dynamics", or the onset of some relatively slower relaxation process. In this line it is tempting to explain the higher $<u^{2}>$ of PSd3 relative to PSd5 in terms of local relaxation processes involving phenyl rings. However, a detailed data analysis shows that relaxation processes can not be the principal source of the observed increase of $\left\langle u^{2}\right\rangle$ beyond the harmonic behavior. First, looking at the time dependence of $S_{\text {exp }}(Q, t, T)$ it is clear that the $<u^{2}>$ obtained from TOF data is dominated by motions in the ps range or even faster. And second, the $\left\langle u^{2}>\right.$ for phenyl-ring hydrogens in PS is larger than that for PSF or PC rings, even though these two polymers, as we will see later, exhibit considerably larger quasielastic scattering due to local relaxation processes of phenyl rings. Therefore, the origin of the departure from the harmonic behavior and the differences in the $<u^{2}>$ s of main-chain and phenyl-ring hydrogens should be looked for in vibrational and "fast-dynamics" type processes rather than in relatively slower and broader local relaxations.

In the frequency $(\omega)$ domain, a significant broadening above the resolution level is found for PSd3 and PSd5 in the TOF window (see figure 2). This increase of quasielastic intensity is higher than that expected due to the enhancement of harmonic vibrations with temperature. If vibrations are accounted for by a Debye-Waller factor and the so-called Bose-Einstein 
scaling procedure, significant quasielastic broadening is still present even well below $\mathrm{T}_{g}$ and in agreement with previous measurements ${ }^{23,24}$. It is likely that most of this quasielastic intensity comes from the first steep decay of the intermediate scattering function at times shorter than $\sim 2 p s$. This phenomenon has been known for a long time in the literature as "fast-dynamics", and its origin together with that of the Boson peak remains an open question. The "fast dynamics" is usually found to set on near $\mathrm{T}_{g}$ and for this reason has often been discussed in terms of the MCT $\beta$ process as a precursor of the glass transition. We note, however, that as previously reported also for other systems ${ }^{23,24,29,30}$ the onset of the "fast dynamics" herein is set on already at temperatures well below $\mathrm{T}_{g}$. Nevertheless, the origin and characterization of this processes is beyond the scope of the present work.

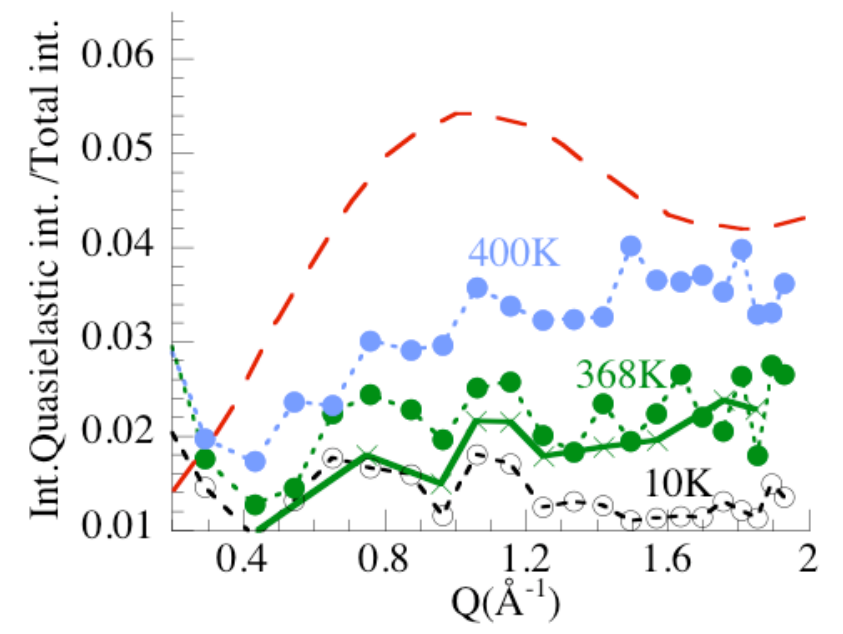

FIG. 8: Integrated quasielastic intensity between 2 and $5 \mu \mathrm{eV}$ in BS window relative to total integrated intensity at 10,368 , and $400 \mathrm{~K}$ for PSd3. Dashed line represents the [1-EISF(Q)] function for a $180^{\circ}$ flip of phenyl rings in arbitrary units. Solid line represent model calculation at $368 \mathrm{~K}$ (see text). 


\section{B. Ring motion in PS by NS}

To characterize the ring motions in PS we have taken advantage of previous experience in other polymers. Thereby, PS's results will be discussed by comparison with previous results on polysulfone (PSF). The scattering of phenyl rings of PSF below its $T_{g}$ will be used here as a model reference system. Phenyl rings in PSF perform fast oscillations of increasing amplitude with temperature and slower $\pi$-flips with an activation energy of $0.43 \mathrm{eV}$. These motions leave representative imprints on the quasielastic neutron scattering from a PSF sample with deuterated methyl groups (PSFd6). The most remarkable are: i) No maxima in the quasielastic intensity of TOF Q-window at $t<2 p s$, due to the small displacement of hydrogens during fast oscillations; ii) a clear maximum in the quasielastic intensity in the BS window at $\mathrm{Q} \sim 1 \AA^{-1}$ and $\mathrm{T} \gtrsim 350 \mathrm{~K}$ due to $\pi$-flips; and iii) a non-trivial amplitude, Qdependence, and T-dependence for the quasielastic and elastic contributions in both TOF and BS windows which narrowly determine the distribution of activation energies corresponding to ring oscillations and flips. We want to note that the results obtained by NS for phenyl-ring motion in PSFd6 nicely matched those observed by other relaxation and NMR techniques.

From the $\mathrm{T}$ and Q-dependence of $A(Q, T)$ in PSd3 we have seen that some dynamic

process enters the $t \gtrsim 2 p s$ TOF window at $\mathrm{T} \gtrsim 200 \mathrm{~K}$, and that this process corresponds to small displacement of hydrogen atoms, as no maximum is observed in the available $\mathrm{Q}$ window. This behavior for the PSd3 dynamics between 2 and 20ps is analogue to that previously observed in PSF, PES, PC or PH, and therefore, it is reasonable to attribute it to small oscillation of phenyl rings. We have performed a quantitative description of the TOF experimental data of PSd3 above 2 ps in terms of phenyl-ring oscillations with T- 
increasing amplitude. The incoherent scattering function for oscillating hydrogen in phenyl rings was

$$
S_{H}^{i n c}(Q, t)=E I S F(Q)+[1-\operatorname{EISF}(Q)] \Phi(t)
$$

where $\Phi(t)$ is the result of a distribution of simple processes having different activation energies,

$$
\Phi(t)=\int \exp \left(-t / \tau\left(E_{a}\right)\right) g\left(E_{a}\right) d E_{a}
$$

and where characteristic times and activation energies are related via an Arrhenius law, $\tau=\tau_{\infty} \cdot \exp \left(E_{a} / K_{B} T\right)$, with $\tau_{\infty}=10^{-13} s$. The $\operatorname{EISF}(\mathrm{Q})$ for ring oscillations was that resulting from a Gaussian distribution of oscillation amplitudes around $0^{\circ}$ of width $\sigma_{\theta}(T)$. As the total coherent scattering cross section and the incoherent scattering cross section $\sigma_{\text {inc }}^{\text {other }}$ of atoms other than hydrogen is much smaller than the incoherent scattering of hydrogen, these contributions have been considered elastic. The model scattering function used to describe data reads as

$$
S(Q, t)=D W F_{\text {eff }}\left[S(Q) \sigma_{\text {coh }}+\sigma_{\text {inc }}^{\text {other }}+\sigma_{\text {inc }}^{H} S_{H}^{\text {inc }}(Q, t)\right]
$$

where $D W F_{\text {eff }}$ is an effective Debye Waller factor accounting for motions faster than 2ps, $\sigma_{c o h}$ and $S(Q)$ are the coherent scattering cross section and the partial static structure factor, and $S_{H}^{\text {inc }}(Q, t)$ is defined by equation 2. During calculations $D W F_{\text {eff }}$ (with $I_{\text {exp }}(Q, t=$ $2 p s)=D W F_{\text {eff }}$ as an input), $\sigma_{\theta}(T)$ and the distribution of activation energies (average and variance) of the oscillation motion were adjusted to best describe the experimental data. Model intermediate scattering functions at different temperatures were normalized to the model $10 \mathrm{~K}$ function for direct comparison with experimental data. The so obtained model intermediate scattering functions are represented by solid lines in figures 5 . The model $\mathrm{A}(\mathrm{Q}, \mathrm{T})$ obtained following the same procedure as with experimental data is also 
represented as a function of $\mathrm{Q}$ and by solid lines in figure 6. The resulting distribution of activation energies for phenyl-ring oscillations is centred at $0.21 \mathrm{eV}$ with a variance of 0.08 $\mathrm{eV}$. This broad distribution suggests that the activation energy would not represent a real intramolecular energy barrier over which phenyl-ring jumps, but would have its origin in rapid fluctuations of the single particle potential. As a comparison, we mention that the oscillation motion of phenyl rings in PSFd6 was characterized by a distribution of activation energies centered at $0.22 \mathrm{eV}$ with a variance of $0.08 \mathrm{eV}$. The similar activation energies for the oscillation motion obtained for PS and PSF rings would indicate that the local surroundings fluctuate with similar characteristic times in both polymers. On the other hand, the amplitude of the oscillation is lower for PS than for PSF or PC $\left(\sigma_{\theta}=24^{\circ}\right.$, $\sigma_{\theta}=28^{\circ}$ and $\sigma_{\theta}=28^{\circ}$ at $368 \mathrm{~K}$ respectively). The lower amplitude for PS suggest a more constrained environment for phenyl rings in PS than for PSF or PC. So, apart form the smaller amplitude, no big differences between side-chain phenyl-ring dynamics in PSd3 and main-chain phenyl-ring dynamics in PSFd6 can be observed at this timescale. In figure 1 the corresponding average characteristic times for the oscillation of phenyl rings in PSd3 have been included by a solid line. Likewise, in the same plot dotted lines represent the limit of characteristic times within the variance of the distribution.

As we have already pointed out, according to the IN6 results the occurrence of generalized $180^{\circ}$ phenyl-ring flips in the TOF window should be ruled out due to the lack of a clear maximum at $Q \sim 1 \AA^{-1}$ in $A(Q, T) / D W F_{\text {eff }}$. Likewise, if generalized slower phenyl-ring $180^{\circ}$ flips should occur in PSd3, a maximum in the intensity of the quasielastic scattering in the BS window at $Q \sim 1 \AA^{-1}$ should be present. However, this is not the case. In the light of these results, NS data support those studies where the fraction of $180^{\circ}$ flipping rings remains small on the timescales covered by TOF and BS measurements, i.e. from $\simeq 10^{-13}$ to $10^{-11} \mathrm{~s}$ 
and from $\simeq 10^{-10}$ to $10^{-9} s$ respectively. It is worth noting that phenyl-ring $180^{\circ}$ flips in PSFd6 are perfectly detectable in the BS window. This implies that either generalized $180^{\circ}$ flips do not occur for phenyl rings in PSd3 or that their timescale is considerably slower than that observed by MDS of PSd3 and by several experimental techniques in phenyl rings of PSF, PC, or PES for example. In this sense, the location of the phenyl rings at the main chain or at a side group seems to have severe implications on their mobility. It should be noted that the experimentally measured quasielastic incoherent scattering is an averaged quantity as it results from the weighted sum of the individual quasielastic incoherent scattering of different phenyl rings. As a consequence, the existence of a very small fraction of $180^{\circ}$ flipping rings can not be excluded, as their signal would be smeared out in the overall averaged signal. On the other hand, The decay of the intermediate scattering function for PSd5 at $t>2 p s$ is even smaller than for PSd3 sample, making a quantitative analysis difficult. From a qualitative point of view, however, it is clear that main-chain hydrogens have very limited mobility in agreement with NMR observations ${ }^{13,14}$.

The contribution of the oscillation motion describing the TOF data at times $t \gtrsim 2 p s$ to the IN16 window has been calculated by convoluting equation 4 in the frequency domain with the experimental resolution. The resulting quasielastic intensity is quite close to that experimentally observed, see figures 8 and 9 . This is an additional indication that oscillations are the main ring motion for PS in the whole dynamic window (BS+TOF) explored here. The agreement between the ring oscillation model and the experiment in BS window is better at high $\mathrm{Q}$ values, where oscillation motions would contribute most, and the ratio of coherent to incoherent scattering is the smallest. On the other hand, the oscillation model underestimates the quasielastic broadening at intermediate Q-values. It is in this Q-range where: i) the coherent contribution would be largest (see Fig 3); and ii) where the influence 
of the small fraction of $180^{\circ}$ flipping rings would be most noticeable, in case this fraction would exist.

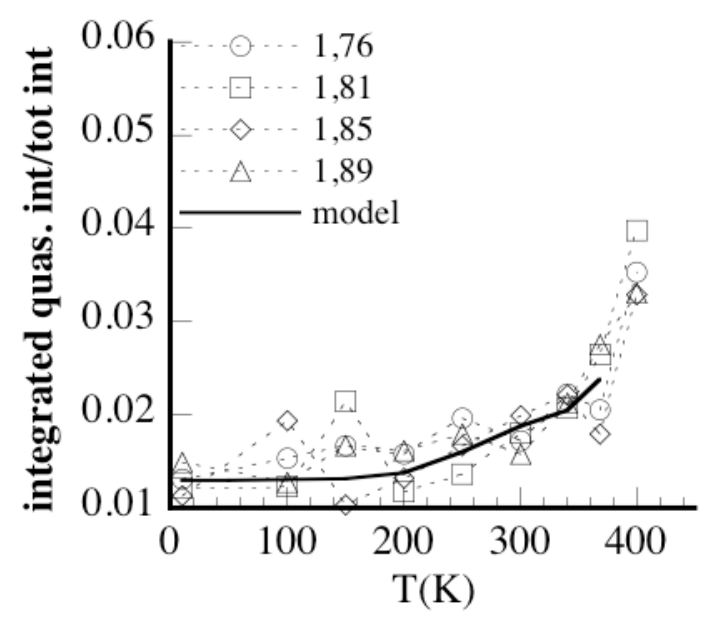

FIG. 9: Integrated quasielastic intensity between 2 and $5 \mu e V$ in BS window relative to total integrated intensity at high Q values (between 1.76 and $1.89 \AA^{-1}$ ) for PSd3 and as a function of T. Solid line represent model calculation (see text).

\section{Secondary relaxations}

Comparing the characteristic times for ring oscillations with the different results of the literature in figure 1 we see that mean ring oscillation time (solid line) is closest to the extrapolated $\delta$-relaxation times, while the times within the slowest wing of the variance for oscillations (dotted line) enter the timescale of the $\gamma$-relaxation and the translational ring motions observed by MDS. At the measured temperatures both $\delta$ and $\gamma$-relaxations are within the timescale covered by the combination of TOF and BS instruments, thereby, our data support that the origin of these relaxations is due to localized low amplitude motions rather than to $180^{\circ}$ flips. As we have already commented in the introduction, the $\gamma$-relaxation has been sometimes related to the $180^{\circ}$ flip motion of phenyl rings. However, the NS results 
presented here show no sign of generalized $180^{\circ}$ phenyl ring flips in glassy PS in the explored NS window. Nevertheless, the presence of $180^{\circ}$ flips of phenyl rings has already been observed by BS at similar temperatures in PSFd6 and other polymeric systems ${ }^{1-4}$, indicating that if $\gamma$-relaxation was due to this type of motion, neutron scattering should be able to detect it. It is worth mentioning that characteristic times for $180^{\circ}$ flips in PSFd6 found by NS match quite well those found by Lyulin et al by MDS for the rotation of phenyl rings in PS. In that work the authors mention that ring rotation was a rare event, so it might be that a very small fraction of phenyl rings in PS are able to flip, whithout producing a measurable imprint in the measured quasielastic signal. Even if the presence of a small fraction of $180^{\circ}$ ring flips can not be excluded, it is very unlikely that this residual motion produces macroscopic mechanic relaxation. PS is known for being a relatively fragile polymer. The lower mobility of phenyl rings in PS in comparison with other polymers having phenyl rings in the main chain follows the idea that only secondary relaxations involving the motion of the main chain are effective for energy dissipation under applied stress. However, we believe that in addition to the position of the ring (in the main chain or as side group), the occurrence of a relatively large amplitude motion of a bulky moiety, like phenyl $\pi$-flips, is also determinant for energy dissipation. Strictly speaking, the $\pi$-flip motion of para-phenyl rings within main chain per se would be irrelevant for mechanic relaxation. Although para-phenyl ring's $\pi$-flip itself cannot be the primary source of mechanic relaxation their close relation is recurrent through the literature. This relation is usually explained in terms of interchain cooperative effects. The argument is that a $\pi$-flip occurs when the separation between two adjacent chains increases, easing steric hindrance and allowing the ring to rotate. In this framework, phenyl-ring motions would activate main-chain motions, and the other way round, explaining the correlation between mechanic relaxation and $\pi$-flip characteristic times. The effect of a 
main-chain ring $\pi$-flip would then propagate through both intra and interchain interactions and extend over a volume far larger than that involved in the rotation itself, facilitating energy dissipation under impact. In the interchain cooperativity scenario explained above, a lower oscillation amplitude would be in agreement with a lower amount of successful $\pi$-flips in PS. Both the weaker intrachain interactions for side-group phenyl rings, and the limited interchain interaction mechanism due to the rare occurrence of $\pi$-flips, would compromise good energy dissipation in PS.

Finally, the mean characterisitic time of the $\beta$-relaxation is very much in the limit of the time window covered by these NS measurements, so that only the high frequency wing of its distribution would be able to produce quasielastic broadening. As a result, little can be said about this relaxation and NS results do not rule out $180^{\circ}$ flip in this longer timescales.

\section{CONCLUSIONS}

NS measurements of partially deuterated PS samples, namely PSd3 and PSd5 have been carried out below the glass transition temperature of the polymers and covering a relatively wide time scale by TOF and BS techniques. Analysis of the elastic intensity and mean square displacement shows that hydrogens in phenyl rings have higher mobility than those in the main chain. The motion of main chain accessed by PSd3 is almost indistinguishable (at least regarding their $<u^{2}>$ ) from that in other simple polymers sequences having $\mathrm{CH}_{2}$ and $\mathrm{CH}$ unities along their main chain structure. The intermediate scattering functions obtained by Fourier transformation of experimental data in the TOF window indicate that the $\left\langle u^{2}>\right.$ is mainly due to motions faster that $\sim 2 p s$, and that secondary relaxations have little effect on its values.

The intermediate scattering functions for PS have the typical two step decay observed 
for other polymeric systems. The magnitude of the second step at $t \gtrsim 2 p s$ is small in the observed window. In the case of PSd3 sample, its T- and Q-dependence is compatible with small displacement of the phenyl hydrogens modeled as oscillations of small amplitude with mean activation energy of $0.21 \mathrm{eV}$. The oscillation of side-chain phenyl rings of PS in the timescale covered by IN6 is similar to that of main-chain phenyl rings in other polymers like PSF or PC. In contrast, quasielastic measurements of PS in BS window show very small broadening above the resolution level and no clear maxima at $Q \sim 1 \AA^{-1}$ which would be indicative of $\pi$-flips of phenyl rings. Almost all the quasielastic broadening observed in PSd3 in the BS window can be well accounted for by the contribution of the oscillation motion inferred from the TOF window to the BS energy window. As a result, it is concluded that below $T_{g}$ generalized $180^{\circ}$ flips do not occur at these timescales for phenyl rings in PSd3. Both $\delta$ and $\gamma$-relaxations are within the timescale covered by the combination of TOF and BS instruments, thereby, our data support that the origin of these relaxations is due to localized low amplitude motions rather than to $180^{\circ}$ flips.

\section{Acknowledgments}

We acknowledge the support of the following research projects, contracts, and grants: DYNACOP PEOPLE-2007-1-1-ITN ref: 214627 by the European Union; MAT-2007-63681 by the Spanish Ministerio de Educación y Ciencia; and IT-436-07 by the Basque Government.

* waaarirs@ehu.es

1 S. Arrese-Igor, A. Arbe, A. Alegría, J. Colmenero, B. Frick. J. Chem. Phys. 120, 423 (2004)

2 S. Arrese-Igor, A. Arbe, A. Alegría, J. Colmenero, B. Frick. J. Chem. Phys. 123, 014907 (2005) 
3 S. Arrese-Igor, A. Arbe, A. Alegría, J. Colmenero, B. Frick. Phys. Rev. E. 75, 051801 (2007)

4 I. Quintana, A. Arbe, J. Colmenero, B. Frick. J. Non-Cryst. Sol. 3524610 (2006)

5 A. F. Yee, S. A. Smith. Macomolecules 14, 54-64 (1981).

6 L. P. Chen, A. F. Yee, S. A. Smith. Macomolecules 32, 5944-5955 (1999).

7 G. P. Johari. J. Chem. Phys. 53, 2372 (1970); G. P. Johari. ibid. 58, 1766 (1973)

8 C. M. Roland, S. Hensel-Bielowka, M. Paluch, and R. Casalini. Rep. Prog. Phys. 68, 1405 $(2005)$

9 O. Rano, Y. Wada. J. Pol. Sci.: part A-2 9669 (1971)

10 J. Schaefer, M. D. Sefcik, E. O. Stejskal, R. A. McKay, W. T. Dixon, R. E. Cais. Macromolecules 17, 669 (1984).

11 A. S. Kulik, K. O. Prins. Polymer 34, 4635 (1993).

12 J. Zhao, Y. H. Chin, Y. Liu, A. A. Jones, P. T. Inglefield, R. P. Kambour, D. M. White. Macromolecules 28, 3889 (1995).

13 M. Vogel, E. Rössler. J. Chem. Phys. 114, 5802 (2001).

14 H. W. Spiess. Coll. and Pol. Sci. 261, 193 (1983).

15 R. F. Rapold, U. W. Suter, D. N. Theodorou. Macromol. Theory Simul. 3, 19 (1994).

16 R. Khare, M. E. Paulaitis. Macomolecules 28, 4495 (1995).

17 A. V. Lyulin, N. K. Balaev, M. A. J. Michels. Macromolecules 35, 9595 (2002).

18 B. Vorselaars, A. V. Lyulin, M. A. J. Michels. Macromolecules 40, 6001 (2007).

19 R. J. Roe. J. Non-Cryst. Sol. 235-237 308 (1998)

20 M. Bée. Physica B 182, 323-336 (1992).

21 T. Kanaya, K. Kaji, K. Inoue. Physica B: Condens. Matt. 180-181, 814 (1992).

22 W. Zajäc, B. Gabrys, D. G. Peiffer, M. A. Adams. Physica B: Condens. Matt. 182, 365 (1992). 
23 B. Frick, U. Buchenau, D. Richter. Colloid Polym. Sci. 276, 413 (1995).

24 T. Kanaya, T. Kawaguchi, K. Kaji. J. Chem. Phys. 104, 5841 (1996).

25 I. Iradi, F. Alvarez, J. Colmenero, A. Arbe. Physica B 350, e881-e884 (2004).

26 R. J. Roe. J. Chem. Phys. 100, 1610 (1993).

27 L. Saviot, E. Duval, A. J. Dianoux, J. F. Jal. Physica B 276-278, 435-436 (2000).

28 R. Pérez-Aparicio, A. Arbe, J. Colmenero, B. Frick, L. Willner, D. Richter, and L. J. Fetters. Macromolecules 39 1060-1072 (2006)

29 U. Buchenau, D. Richter. Phys. Rev. Lett. 73, 2344 (1994).

30 S. Arrese-Igor, I. Quintana, A. Arbe, J. Colmenero, A. Alegría, B. Frick, S. Janssen. Physica B. 350, e971 (2004)

31 We limited the integrating window to an area close to the elastic peak instead of taking the full quasielastic range in order to minimize the effect of the contribution of fast oscillations centred at IN6 on the observed Q-dependence. 


\section{FIGURE CAPTIONS}

Figure 1: Characteristic times map for different dynamic processes in PS measured by different techniques: (i) Mechanic and dielectric relaxations, filled and empty diamonds respectively $^{9}$; (ii) NMR, up-triangles ${ }^{12}$, empty circles ${ }^{11}$ and shadowed rectangular areas ${ }^{10}$; (iii) MDS, solid circles, solid and half-solid squares, and right-triangles ${ }^{17}$; and (iv) NS this work, solid and dotted lines. Dashed lines represent fits of data in the literature to Arrhenius laws.

Figure 2: PSd5 (panel a) and PSd3 (panel b) spectra measured by IN6-TOF at $\mathrm{Q}=1.37 \AA^{-1}$ and at different temperatures 10, 100 (only PSd3), 200, 250, 300,340 and $368 \mathrm{~K}$ from bottom to top. Inset: detail of the elastic intensity.

Figure 3: Elastic intensity of PSd5 (panel a) and PSd3 (panel b) in TOF window normalized to $10 \mathrm{~K}$ and $13 \mathrm{~K}$ values respectively. Solid lines represent fittings to $\propto \exp \left[-<u^{2}>\right.$ $\left.Q^{2} / 3\right]$. Crosses represent the ratio of coherent to total intensity measured by diffraction measurements at $\mathrm{D} 7^{25}$

Figure 4: Mean squared displacement as a function of temperature for PSd5 (squares) and PSd3 (circles) obtained from the elastic maxima of the spectra in TOF window (see text). Solid lines represent fits to an harmonic law at $T \leq 150 \mathrm{~K}$. Plusses and crosses represent the $\left\langle u^{2}>\right.$ of Polyethylenepropylene and Head to head Polypropylene respectively ${ }^{28}$. Inset: T-dependence of the elastic intensity normalized to 10 (PSd3) and 13K (PSd5) values at $Q=1.64 \AA^{-1}$.

Figure 5: Intermediate scattering functions for PSd3 obtained by Fourier transform of experimental data measured on IN6 at $\mathrm{Q}=1.37 \AA^{-1}$ and at the temperatures indicated. Lines represent calculated intermediate scattering functions for the oscillation model (see text). Vertical line marks $t=2$ ps. 
Figure 6: $A(Q, T)$ and $A(Q, T) / D W F_{\text {eff }}$ for PSd3 in TOF window as a function of $\mathrm{T}$ panel a)and Q panel b) respectively. Solid lines represent model calculations (see text). Although the amplitude of the motion is small, it does not show the typical $Q^{2}$ dependence because the model EISF was that resulting from a Gaussian distribution of amplitudes.

Figure 7: Normalized scattering functions $I_{\exp }(Q, \omega, T)$ at $Q=1.64 \AA^{-1}$ for PSd3 at $368 \mathrm{~K}$ (panel a) and $400 \mathrm{~K}$ (panel b), and for PSd5 at $368 \mathrm{~K}$ (panel c). Lines stand for measurements at $10 \mathrm{~K}$ representing the resolution of the instrument. Empty symbols in panel b represent data for PSFd6 at $350 \mathrm{~K}$ measured at the same instrument and configuration

Figure 8: Integrated quasielastic intensity between 2 and $5 \mu \mathrm{eV}$ in BS window relative to total integrated intensity at 10,368 , and $400 \mathrm{~K}$ for PSd3. Dashed line represents the [1-EISF(Q)] function for a $180^{\circ}$ flip of phenyl rings in arbitrary units. Solid line represent model calculation at $368 \mathrm{~K}$ (see text).

Figure 9: Integrated quasielastic intensity between 2 and $5 \mu \mathrm{eV}$ in BS window relative to total integrated intensity at high Q values (between 1.76 and $1.89 \AA^{-1}$ ) for PSd3 and as a function of T. Solid line represent model calculation (see text). 


\section{FOR TABLE OF CONTENTS USE ONLY}

GLASSY DYNAMICS OF POLYSTYRENE BY QUASIELASTIC NEUTRON SCATTERING

Silvia Arrese-Igor, Arantxa Arbe, Bernhard Frick, and Juan Colmenero

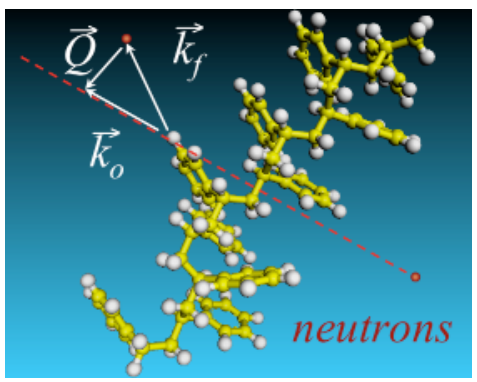

